\title{
Certain sufficient conditions for strongly starlikeness and convexity
}

\author{
Yu-Qin Tao' and Jin-Lin Liư ${ }^{*}$
}

"Correspondence: jliu@yzu.edu.cn 2Department of Mathematics, Yangzhou University, Yangzhou, 225002, China

Full list of author information is available at the end of the article

\begin{abstract}
The object of the present paper is to derive some sufficient conditions for strongly starlikeness and convexity.

MSC: $30 C 45$

Keywords: analytic function; starlike function; convex function; strongly starlike function; strongly convex function
\end{abstract}

\section{Introduction}

Let $A(n)(n \geq 2)$ denote the class of functions $f(z)$ of the form

$$
f(z)=z+\sum_{k=n}^{\infty} a_{n} z^{n}
$$

which are analytic in the open unit disc $U=\{z:|z|<1\}$. We write $A=A(2)$. Let $S^{*}$ and $K$ be the subclasses of $A(n)$ consisting of all starlike functions $f(z)$ in $U$ and of all convex functions $f(z)$ in $U$, respectively.

$$
\begin{aligned}
& \text { If } f(z) \in A(n) \text { satisfies } \\
& \qquad\left|\arg \left(\frac{z f^{\prime}(z)}{f(z)}\right)\right|<\frac{\pi}{2} \gamma \quad(z \in U)
\end{aligned}
$$

for some $\gamma(0<\gamma \leq 1)$, then $f(z)$ is said to be strongly starlike of order $\gamma$ in $U$, and denoted by $f(z) \in \widetilde{S^{*}}(\gamma)$. If $f(z) \in A(n)$ satisfies

$$
\left|\arg \left(1+\frac{z f^{\prime \prime}(z)}{f^{\prime}(z)}\right)\right|<\frac{\pi}{2} \gamma \quad(z \in U)
$$

for some $\gamma(0<\gamma \leq 1)$, then we say that $f(z)$ is strongly convex of order $\gamma$ in $U$, and we denote by $\widetilde{K}(\gamma)$ the class of all such functions. It is obvious that $f(z) \in A(n)$ belongs to $\widetilde{K}(\gamma)$ if and only if $z f^{\prime}(z) \in \widetilde{S^{*}}(\gamma)$. Further, we note that $\widetilde{S^{*}}(1)=S^{*}$ and $\widetilde{K}(1)=K$.

The strongly starlike and convex functions have been extensively studied by several authors (see, e.g., [1-11]). The object of the present paper is to derive some sufficient conditions for strongly starlikeness and strongly convexity. Some previous results are extended.

\section{盟 Springer}

(c) 2013 Tao and Liu; licensee Springer. This is an Open Access article distributed under the terms of the Creative Commons Attribution License (http://creativecommons.org/licenses/by/2.0), which permits unrestricted use, distribution, and reproduction in any medium, provided the original work is properly cited. 
For our purpose, we have to recall here the following results.

Lemma 1 (see [5]) Let a function $p(z)=1+c_{1} z+c_{2} z^{2}+\cdots$ be analytic in $U$ and $p(z) \neq 0$ $(z \in U)$. If there exists a point $z_{0} \in U$ such that

$$
|\arg p(z)|<\frac{\pi}{2} \beta \quad\left(|z|<\left|z_{0}\right|\right)
$$

and

$$
\left|\arg p\left(z_{0}\right)\right|=\frac{\pi}{2} \beta \quad(0<\beta \leq 1)
$$

then

$$
\frac{z_{0} p^{\prime}\left(z_{0}\right)}{p\left(z_{0}\right)}=i k \beta
$$

where

$$
\begin{aligned}
& k \geq \frac{1}{2}\left(a+\frac{1}{a}\right) \quad\left(\text { when } \arg p\left(z_{0}\right)=\frac{\pi}{2} \beta\right), \\
& k \leq-\frac{1}{2}\left(a+\frac{1}{a}\right) \quad\left(\text { when } \arg p\left(z_{0}\right)=-\frac{\pi}{2} \beta\right),
\end{aligned}
$$

and $p\left(z_{0}\right)^{1 / \beta}= \pm i a(a>0)$.

Lemma 2 (see [4]) If $f(z) \in A$ satisfies

$$
\left|f^{\prime}(z)-1\right|<\frac{\sqrt{20}}{5} \quad(z \in U)
$$

then $f(z) \in S^{*}$.

\section{Starlikeness and convexity}

Our first result is contained in the following.

Theorem 1 Let $0<\alpha \leq \frac{1}{1+\frac{2}{\pi} \int_{0}^{1} \sin ^{-1}\left(\frac{2 \rho}{1+\rho^{2}}\right) d \rho}$. If $f(z) \in A(n)(n \geq 2)$ satisfies

$$
\left|\arg f^{\prime}(z)\right|<\frac{\pi}{2} \alpha \quad(z \in U)
$$

then $f(z) \in \widetilde{S^{*}}(\beta)$, where

$$
\beta=\left(1+\frac{2}{\pi} \int_{0}^{1} \sin ^{-1}\left(\frac{2 \rho}{1+\rho^{2}}\right) d \rho\right) \alpha .
$$

Proof Note that

$$
\arg f^{\prime}(z)=\arg \left(\frac{z f^{\prime}(z)}{f(z)}\right)+\arg \left(\frac{f(z)}{z}\right)
$$


and

$$
\begin{aligned}
\arg \left(\frac{f(z)}{z}\right) & =\arg \left(\frac{1}{z} \int_{0}^{z} f^{\prime}(t) d t\right) \\
& =\arg \left(\frac{1}{z} \int_{0}^{r} f^{\prime}\left(\rho e^{i \theta}\right) e^{i \theta} d \rho\right) \quad\left(z=r e^{i \theta}, t=\rho e^{i \theta}\right) \\
& =\arg \left(\int_{0}^{r} f^{\prime}\left(\rho e^{i \theta}\right) d \rho\right) .
\end{aligned}
$$

Let

$$
0=\rho_{0}<\rho_{1}<\rho_{2}<\cdots<\rho_{m-1}<\rho_{m}=r,
$$

and

$$
\rho_{j}-\rho_{j-1}=\delta_{m} \quad(j=1,2, \ldots, m) .
$$

Then, by using (2.2), we have that

$$
\left|\arg \left(\frac{f(z)}{z}\right)\right|=\left|\arg \left(\lim _{m \rightarrow \infty} \sum_{j=1}^{m} \delta_{m} f^{\prime}\left(\rho_{j} e^{i \theta}\right)\right)\right| \leq \lim _{m \rightarrow \infty} \sum_{j=1}^{m} \delta_{m}\left|\arg f^{\prime}\left(\rho_{j} e^{i \theta}\right)\right| .
$$

Since the condition (2.1) implies that

$$
f^{\prime}(z) \prec\left(\frac{1+z}{1-z}\right)^{\alpha} \quad(z \in U),
$$

we obtain that

$$
\begin{aligned}
\left|\arg \left(\frac{f(z)}{z}\right)\right| & \leq \lim _{m \rightarrow \infty} \sum_{j=1}^{m} \delta_{m}\left|\arg \left(\frac{1+\rho_{j} e^{i \theta}}{1-\rho_{j} e^{i \theta}}\right)^{\alpha}\right|<\alpha \int_{0}^{r} \sin ^{-1}\left(\frac{2 \rho}{1+\rho^{2}}\right) d \rho \\
& <\alpha \int_{0}^{1} \sin ^{-1}\left(\frac{2 \rho}{1+\rho^{2}}\right) d \rho \\
& =\frac{\pi}{2} \alpha\left(\frac{2}{\pi} \int_{0}^{1} \sin ^{-1}\left(\frac{2 \rho}{1+\rho^{2}}\right) d \rho\right) .
\end{aligned}
$$

Furthermore, since

$$
\left|\arg \left(\frac{z f^{\prime}(z)}{f(z)}\right)\right|-\left|\arg \left(\frac{f(z)}{z}\right)\right| \leq\left|\arg f^{\prime}(z)\right| \quad(z \in U),
$$

we conclude from (2.1) and (2.3) that

$$
\begin{aligned}
\left|\arg \left(\frac{z f^{\prime}(z)}{f(z)}\right)\right| & \leq\left|\arg f^{\prime}(z)\right|+\left|\arg \left(\frac{f(z)}{z}\right)\right|<\frac{\pi}{2} \alpha+\frac{\pi}{2} \alpha\left(\frac{2}{\pi} \int_{0}^{1} \sin ^{-1}\left(\frac{2 \rho}{1+\rho^{2}}\right) d \rho\right) \\
& =\frac{\pi}{2} \beta,
\end{aligned}
$$

which shows that $f(z) \in \widetilde{S^{*}}(\beta)$. 
Theorem 2 Let $0<\alpha \leq 1$. Iff $(z) \in A(n)(n \geq 2)$ satisfies

$$
\left|\arg \left(f^{\prime}(z)+z f^{\prime \prime}(z)\right)\right|<\frac{\pi}{2} \alpha\left(\alpha_{1}+\frac{2}{\pi} \tan ^{-1} \alpha_{1}\right) \quad(z \in U),
$$

then $f(z) \in \widetilde{K}(\alpha)$, where $\alpha_{1}=0.3834 \ldots$ is the root of the equation

$$
2 \alpha_{1}+\frac{2}{\pi} \tan ^{-1} \alpha_{1}=1
$$

Proof Note that

$$
\arg \left(f^{\prime}(z)+z f^{\prime \prime}(z)\right)=\arg f^{\prime}(z)+\arg \left(1+\frac{z f^{\prime \prime}(z)}{f^{\prime}(z)}\right) .
$$

If there exists a point $z_{0} \in U$ such that

$$
\left|\arg f^{\prime}(z)\right|<\frac{\pi}{2} \alpha_{1} \alpha \quad\left(|z|<\left|z_{0}\right|\right)
$$

and

$$
\left|\arg f^{\prime}\left(z_{0}\right)\right|=\frac{\pi}{2} \alpha_{1} \alpha
$$

then by Lemma 1, we have

$$
\frac{z_{0} f^{\prime \prime}\left(z_{0}\right)}{f^{\prime}\left(z_{0}\right)}=i \alpha_{1} \alpha k
$$

Therefore, if $\arg f^{\prime}\left(z_{0}\right)=\frac{\pi}{2} \alpha_{1} \alpha$, then we have

$$
\begin{aligned}
\arg f^{\prime}\left(z_{0}\right)+\arg \left(1+\frac{z_{0} f^{\prime \prime}\left(z_{0}\right)}{f^{\prime}\left(z_{0}\right)}\right) & =\frac{\pi}{2} \alpha_{1} \alpha+\arg \left(1+i \alpha_{1} \alpha k\right) \\
& =\frac{\pi}{2} \alpha_{1} \alpha+\tan ^{-1}\left(\alpha_{1} \alpha k\right) \\
& \geq \frac{\pi}{2} \alpha_{1} \alpha+\alpha \tan ^{-1} \alpha_{1} \\
& =\frac{\pi}{2} \alpha\left(\alpha_{1}+\frac{2}{\pi} \tan ^{-1} \alpha_{1}\right),
\end{aligned}
$$

which contradicts (2.4). If $\arg f^{\prime}\left(z_{0}\right)=-\frac{\pi}{2} \alpha_{1} \alpha$, then applying the same method for the previous case, we also have

$$
\arg f^{\prime}\left(z_{0}\right)+\arg \left(1+\frac{z_{0} f^{\prime \prime}\left(z_{0}\right)}{f^{\prime}\left(z_{0}\right)}\right) \leq-\frac{\pi}{2} \alpha\left(\alpha_{1}+\frac{2}{\pi} \tan ^{-1} \alpha_{1}\right)
$$

which contradicts (2.4). Therefore, there exists no $z_{0} \in U$ such that $\left|\arg f^{\prime}\left(z_{0}\right)\right|=\frac{\pi}{2} \alpha_{1} \alpha$. This implies that

$$
\left|\arg f^{\prime}(z)\right|<\frac{\pi}{2} \alpha_{1} \alpha \quad(z \in U) .
$$


Furthermore, since

$$
\begin{aligned}
\left|\arg \left(1+\frac{z f^{\prime \prime}(z)}{f^{\prime}(z)}\right)\right|-\left|\arg f^{\prime}(z)\right| & \leq\left|\arg \left(f^{\prime}(z)+z f^{\prime \prime}(z)\right)\right| \\
& <\frac{\pi}{2} \alpha\left(\alpha_{1}+\frac{2}{\pi} \tan ^{-1} \alpha_{1}\right) \quad(z \in U),
\end{aligned}
$$

we conclude that

$$
\left|\arg \left(1+\frac{z f^{\prime \prime}(z)}{f^{\prime}(z)}\right)\right|<\frac{\pi}{2} \alpha\left(2 \alpha_{1}+\frac{2}{\pi} \tan ^{-1} \alpha_{1}\right)=\frac{\pi}{2} \alpha \quad(z \in U),
$$

which shows that $f(z) \in \widetilde{K}(\alpha)$.

Theorem 3 Iff $(z)=z+a_{n} z^{n}+\cdots \in A(n)(n \geq 2)$ satisfies

$$
\left|f^{(n)}(z)\right| \leq \frac{\sqrt{20}}{5} \quad(z \in U)
$$

then $f(z) \in S^{*}$.

Proof From (2.5), one can see that

$$
\begin{aligned}
\left|f^{(n-1)}(z)\right| & =\left|\int_{0}^{z} f^{(n)}(t) d t\right| \\
& \leq \int_{0}^{|z|}\left|f^{(n)}(t)\right||d t| \\
& \leq \frac{\sqrt{20}}{5}|z|<\frac{\sqrt{20}}{5} \quad(z \in U), \\
\ldots & \\
f^{\prime \prime}(z) \mid \leq & \frac{\sqrt{20}}{5} \quad(z \in U) .
\end{aligned}
$$

Noting that

$$
\begin{aligned}
\left|f^{\prime}(z)-1\right| & =\left|\int_{0}^{z} f^{\prime \prime}(t) d t\right| \\
& \leq \int_{0}^{|z|}\left|f^{\prime \prime}(t)\right||d t| \\
& \leq \frac{\sqrt{20}}{5}|z|<\frac{\sqrt{20}}{5} \quad(z \in U) .
\end{aligned}
$$

By Lemma 2, we have $f(z) \in S^{*}$.

Theorem 4 Iff $(z)=z+a_{n} z^{n}+\cdots \in A(n)(n \geq 2)$ satisfies

$$
\left|f^{(n)}(z)\right| \leq \frac{\sqrt{5}}{5} \quad(z \in U)
$$

then $f(z) \in K$. 
Proof By using the same method as in the proof of Theorem 3, we have

$$
\left|f^{\prime \prime}(z)\right| \leq \frac{\sqrt{5}}{5} \quad(z \in U)
$$

It follows that

$$
\begin{aligned}
\left|\left(z f^{\prime}(z)\right)^{\prime}-1\right| & =\left|f^{\prime}(z)+z f^{\prime \prime}(z)-1\right| \\
& \leq\left|f^{\prime}(z)-1\right|+\left|z f^{\prime \prime}(z)\right| \\
& \leq\left|\int_{0}^{z} f^{\prime \prime}(t) d t\right|+\left|z f^{\prime \prime}(z)\right| \\
& \leq \int_{0}^{|z|}\left|f^{\prime \prime}(t)\right||d t|+\frac{\sqrt{5}}{5}|z| \\
& \leq \frac{2 \sqrt{5}}{5}|z|<\frac{\sqrt{20}}{5} \quad(z \in U) .
\end{aligned}
$$

Therefore, using Lemma 2, we see that $z f^{\prime}(z) \in S^{*}$, or $f(z) \in K$.

\section{Competing interests}

The authors declare that they have no competing interests.

\section{Authors' contributions}

The authors have made the same contribution. All authors read and approved the final manuscript.

\section{Author details}

${ }^{1}$ Department of Mathematics, Maanshan Teacher's College, Maanshan, 243000, China. ${ }^{2}$ Department of Mathematics, Yangzhou University, Yangzhou, 225002, China.

\section{Acknowledgements}

Dedicated to Professor Hari M Srivastava.

We would like to express sincere thanks to the referees for careful reading and suggestions which helped us to improve the paper.

\section{Received: 16 January 2013 Accepted: 25 March 2013 Published: 10 April 2013}

\section{References}

1. Gangadharan, A, Ravichandran, V: Radii of convexity and strong starlikeness for some classes of analytic functions. J. Math. Anal. Appl. 211, 303-313 (1997)

2. Liu, J-L: The Noor integral operator and strongly starlike functions. J. Math. Anal. Appl. 261, 441-447 (2001)

3. Liu, J-L: Certain sufficient conditions for strongly starlike functions associated with an integral operator. Bull. Malays. Math. Soc. 34, 21-30 (2011)

4. Mocanu, PT: Some starlikeness conditions for analytic functions. Rev. Roum. Math. Pures Appl. 33, 117-124 (1988)

5. Nunokawa, M: On the order of strongly starlikeness of strongly convex functions. Proc. Jpn. Acad., Ser. A, Math. Sci. 68, 234-237 (1993)

6. Nunokawa, M, Owa, S, Polatoglu, Y, Caglar, M, Duman, EY: Some sufficient conditions for starlikeness and convexity. Turk. J. Math. 34, 333-337 (2010)

7. Nunokawa, M, Owa, S, Saitoh, H, Ikeda, A, Koike, N: Some results for strongly starlike functions. J. Math. Anal. Appl. 212, 98-106 (1997)

8. Nunokawa, M, Thomas, DK: On convex and starlike functions in a sector. J. Aust. Math. Soc. A 60, $363-368$ (1996)

9. Obradovic, M, Owa, S: Some sufficient conditions for strongly starlikeness. Int. J. Math. Math. Sci. 24, 643-647 (2000)

10. Ponnusamy, S, Singh, V: Criteria for strongly starlike functions. Complex Var. Theory Appl. 34, 267-291 (1997)

11. Xu, N, Yang, D-G, Owa, S: On strongly starlike multivalent functions of order $\beta$ and type $\alpha$. Math. Nachr. 283, 1207-1218(2010) 